\title{
Evaluation of Ventricular Tachycardia with Respect to Syncope in Patients with Old Myocardial Infarction, Dilated Cardiomyopathy and No Overt Heart Disease
}

\author{
Teruhisa Tanabe, M.D. and Yuichiro Goto, M.D.
}

\begin{abstract}
The incidence and the direct cause of syncope in ventricular tachycardia (VT) among patients with old myocardial infarction (OMI, $n=48)$, dilated cardiomyopathy (DCM, $n=18$ ) and no evidence of heart disease (IVT, $n=43$ ) were compared. The presence or absence of syncope in each patient was surveyed by a standardized questionaire and a variety of electrocardiographic parameters for aggravating arrhythmias were measured. Syncope occurred in 19 of 43 OMI patients $(40 \%)$, in 5 of 18 DCM patients $(28 \%)$ and 6 of 43 IVT patients $(14 \%)$ and significantly more often in OMI than IVT $(\mathrm{p}<0.01)$. Ventricular fibrillation (VF) was confirmed in 14 of the 19 OMI patients with syncope, in 3 of the 5 DCM patients with syncope and 1 of 6 IVT patients with syncope. The incidence of VF was significantly higher in OMI than in IVT $(\mathrm{p}<0.01)$. Mean VT cycle lengths (VTRR'm) in OMI patients with and without syncope were $0.35 \pm 0.07 \mathrm{sec}$ and $0.42 \pm 0.10 \mathrm{sec}$, respectively $(\mathrm{p}<0.05)$. VTRR'ms in DCM patients with and without syncope were $0.43 \pm 0.10 \mathrm{sec}$ and $0.42 \pm 0.10 \mathrm{sec}$, respectivrly (NS). VTRR'ms in IVT patients with and without syncope were $0.27 \pm 0.04 \mathrm{sec}$ and $0.41 \pm 0.10 \mathrm{sec}$, respectively $(\mathrm{p}<0.01)$. The results show that the high frequency of VT rate was the main cause of syncope in IVT, while VF was the main cause of syncope in OMI. There were no significant differences in the coupling interval, prematurity index, vulnerability index and QTc between groups with and without syncope in any of the underlying diseases. There were also no significant differences in the ejection fraction between the 2 groups.

Therefore, it is considered that there is a diffrence in the direct cause of syncope between VT patients with OMI and IVT. Impaired ventricular function was not an important factor of syncope. Both VF and rapid VT are the main causes of syncope in OMI, while rapid VT is the main cause of syncope in IVT. This may be one of the reasons why IVT patients generally do not experience sudden cardiac death.
\end{abstract}

$\mathbf{S}$ UDDEN cardiac death or syncope is one of the major problems in patients with ventricular tachycardia (VT). VTs occur not only in patients with underlying heart disease but also in those with no evidence of heart disease (idiopathic VT: IVT). The

Key words:

Ventricular tachycardia VT

Rapidity of VT

Syncope

Organic heart disease

Idiopathic VT former VTs reportedly have high incidences of sudden cardiac death ${ }^{1-3}$ whereas the latter ones have low or no incidence of sudden cardiac death, ${ }^{4-6}$ although the reason remains unknown.

Syncope may be a possible prodrome of sudden cardiac death in VT patients with underlying disease ${ }^{7-9}$ However, it may result in very few case of sudden cardiac death in IVT patients, as proven by previous studies. 6

In order to clarify some of the differ-

Department of Cardiology, Tokai University School of Medicine, Bohseidai, Isehara, Japan

Mailing address: Teruhisa Tanabe, M.D., Department of Cardiology, Tokai University School of Medicine, Bohseidai, Isehara, 259-11, Japan 


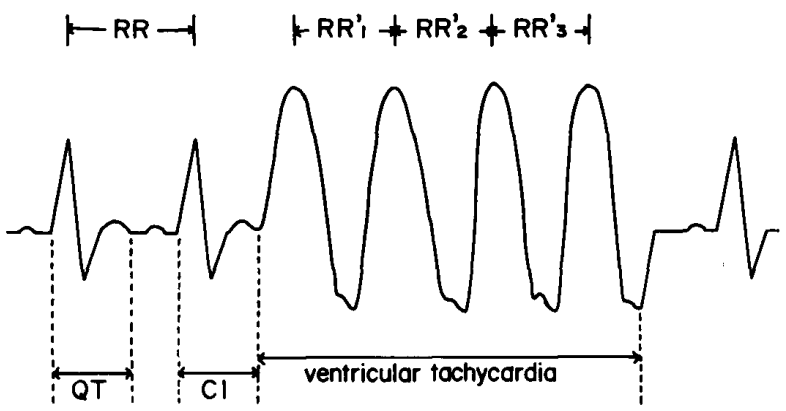

Fig.1. Electrocardiographic parameters measured in this study.

ences, the incidences of syncope and ventricular fibrillation (VF), and ejection fraction (EF) were compared among VT patients with old myocardial infarction (OMI), those with dilated cardiomyopathy (DCM) and those with IVT. In addition, electrocardiographic parameters, which are often used to predict the occurrence of life-threatening arrhythmias associated with sudden cardiac death or loss of consciousness, were also compared among the 3 underlying diseases mentioned above.

\section{SUBJECTS AND METHODS}

Study patients

A total of 109 patients with OMI
Diagnosis and Treatment of Life-threatening Arrhythmia

$(n=48)$, DCM $(n=18)$ and IVT $(n=43)$ were enrolled in this study. All patients had at least 3 or more VTs (runs of more than 3 consecutive ventricular contractions at a minimum rate of $120 \mathrm{bpm}$ ) per day in the recordings obtained from Holter monitoring or conventional heart monitor.

The patients in OMI, DCM and IVT groups ranged in age from 40 to 89 years $(\mathrm{m} \pm \mathrm{SD}, 63.1 \pm 11.9)$, from 18 to 66 years $(49.7 \pm 15.1)$, and from 16 to 70 years (48.8 \pm 14.7$)$, respectively. Patients with acute or recent myocardial infarction, unstable angina pectoris, deteriorating congestive heart failure, advanced A-V block, sick sinus syndrome, electrolyte imbalance, hypoxia or digitals intoxication were excluded from the study. Presence or absence of syncope was surveyed from a direct interview with each patient, or from a telephone interview with a witness as to loss of consciousness, or from the doctor's description of patient hospitalization.

Syncope was defined as a sudden, transient loss of consciousness associated with an inability to maintain posture followed by spontaneous recovery over seconds to minutes. Loss of consciousness caused by seizures, vertigo, semicoma, coma, shock and other conditions of unconsciousness unre-

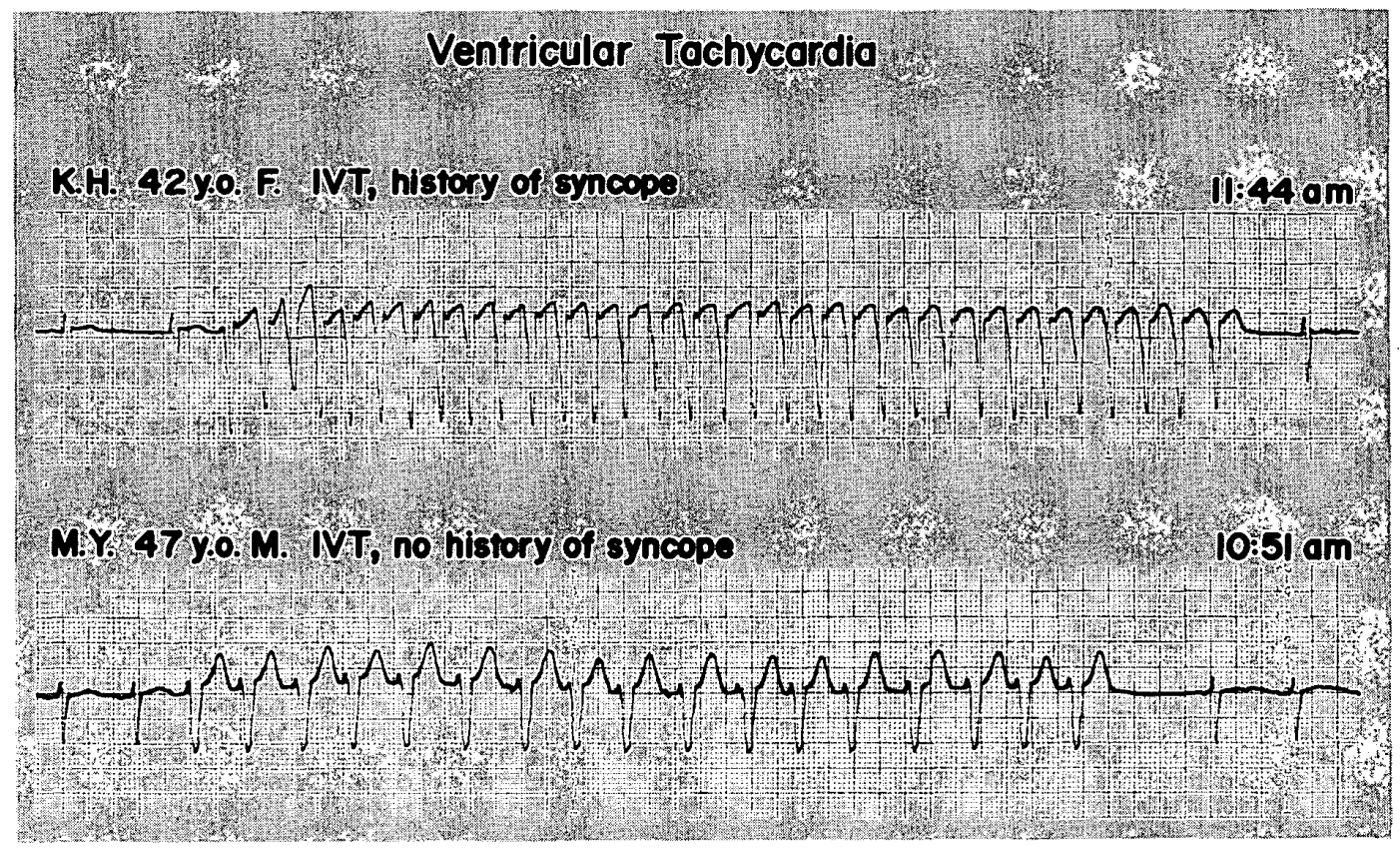

Fig.2. Holter strips showing VT in two different patients with idiopathic VT. The electrocardiogram in the top shows very rapid VT, while that in the bottom demonstrates non-rapid VT. 


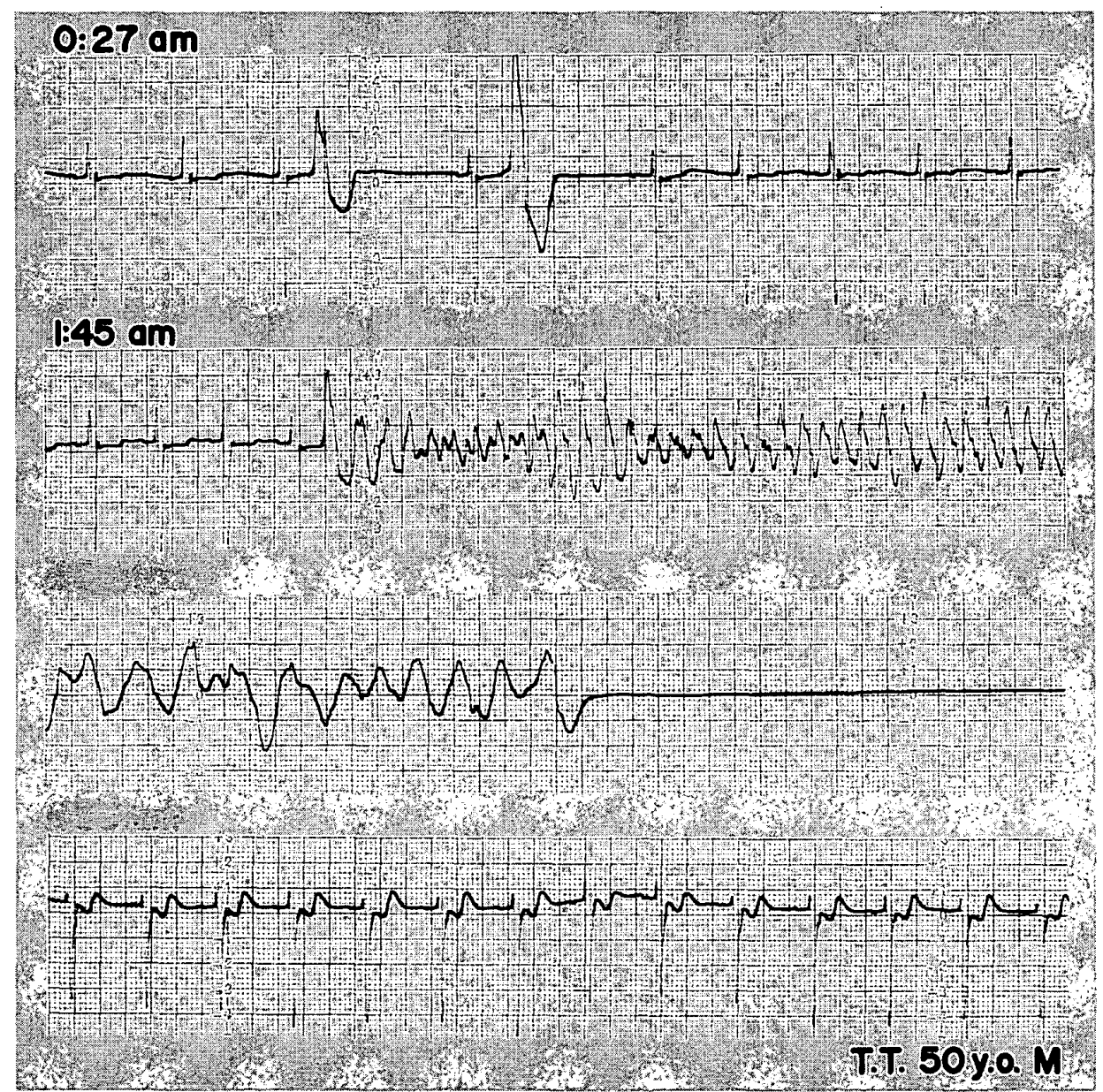

Fig.3. Holter strips showing $\mathrm{R}$ on $\mathrm{T}$ ventricular premature beats (top), a burst of torsade de pointes VT following a $\mathrm{R}$ on $\mathrm{T}$ ventricular premature beat (the second panel), ventricular fibrillation followed by cardiac arrest (the third panel) and recovery of sinus beats (bottom) in a 50-year old male patient with angina pectoris.

lated to heart problems were excluded. When electrocardiograms were recorded during the documentation of syncope, which occurred during hospitalization or upon admission to the emergency room, the presence or absence of VF was surveyed.

\section{Recordings and analysis of Holter ECGs}

A Del Mar-Avionics Electrocardiocorder 447 was used for the Holter monitoring. Each tape was analyzed using a Del MarAvionics Dynamic Electrocardioscanner DCG VII and a Fukuda-Denshi-Co sigmacorder, which could print out a electrocardiogram of 24 consecutive hours in $12 \mathrm{~min}$.

The longest VT (VT max) in each Holter monitoring was detected from the recording of a sigmacorder and the recording of the real-time speed of ECG $(2.5 \mathrm{~cm} / \mathrm{sec})$ was made on the VT max. The following parameters of VT max were measured (Fig. 1):

1. the RR interval of sinus beat preceding the VT max (RR).,

2 . the coupling interval $(\mathrm{CI})$ of ventricular premature beat initiating VT max.,

3. prematurity index (PI, CI/QT) and vulnerability index (VI, RR/PI). ${ }^{10}$

4. QTc $(\mathrm{QT} / \sqrt{\mathrm{RR}})$.,

5. VTRR'm which reflected the mean cycle length of each VT max: $\mathrm{RR}_{1}{ }_{1}+\mathrm{RR}_{2}{ }_{2}+$ $\mathrm{RR}^{\prime---}+\mathrm{RR}$ 'n/n.,

6. SDRR'm which means the standard deviation of each VTRR'm.

VTRR'ms and SDRR'ms were measured in order to compare the variability of cycle length of VT max among patients with OMI, DCM and IVT, and to compare it with and without syncope. 


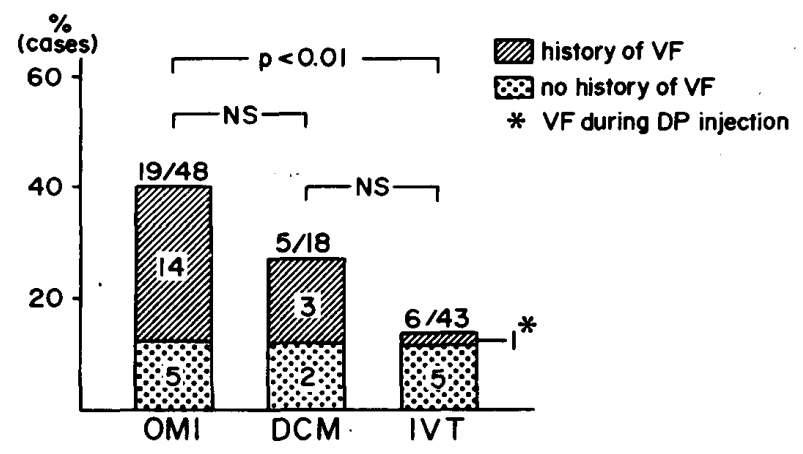

Fig.4. Comparison of the number of patients with syncope among old myocardial infarction (OMI), dilated cardiomyopathy (DCM) and idiopathic ventricular tachycardia (IVT). Striped and dotted areas in each column reflect the number of patient with and without ventricular fibrillation (VF), respectively.

\section{Measurement of ejection fraction}

EFs; determined by radionuclide ventriculography, were compared among the patients with and without syncope in order to determine whether or not left ventricular dysfunction influenced the occurrence of syncope.

\section{RESULTS}

Figure 2 reveals Holter strips showing VT of 2 patients with idiopathic VT. Each patient had frequent episodes of VT on the same Holter monitoring and almost the same cycle length of VT at any VT. However, cycle length of VT was quite diffrent between the two patients. The patient in the top panel showed very rapid VT as compared to the one in the bottom panel. The patient in the top panel had several episodes of syncope, which also occurred the day before Holter monitoring was done. In contrast the patient in the bottom panel, who showed non-rapid VT, had never experienced syncope or syncope-like symptoms.

Figure 3 demonstrates Holter strips showing $\mathrm{R}$ on $\mathrm{T}$ ventricular premature beats (top), a burst of torsade de pointes tachycardia following a $\mathrm{R}$ on $\mathrm{T}$ ventricular premature beat (the second panel), VF followed by cardiac arrest (the third panel) and recovery of sinus beats (bottom). This 50 -year old man with angina pectoris had serious ventricular arrhythmias as mentioned in

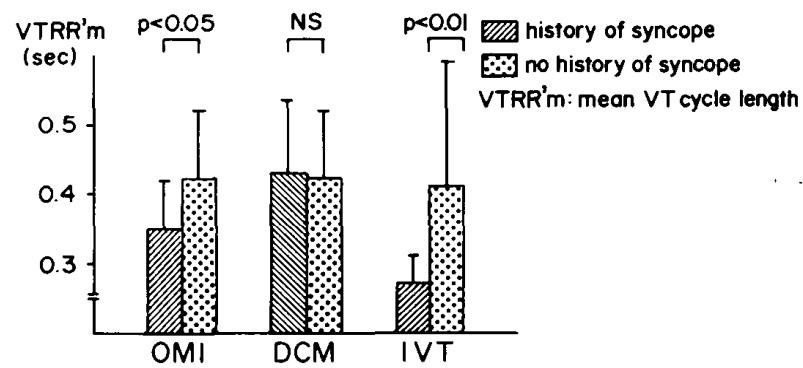

Fig.5. Comparison of the mean VT cycle lenght (VTRR'm) between groups with and without syncope in old myocardial infarction (OMI), dilated cardiomyopathy (DCM) and idiopathic ventricular tachycardia (IVT).

Fig.3, and loss consciousness, which occurred during Holter monitoring.

From these observations, it seems that there are two kinds of ventricular arrythmias directly associated with cardiac unconsciousness: the very rapid VT and VF itself. Some VFs followed a torsade de pointes tachycardia and the others were preceded by non-fast VT.

Figure 4 shows the comparison of the number of patients with syncope among OMI, DCM and IVT. Striped areas in each column reflect the number of patients with confirmation of $\mathrm{VF}$, while dotted areas reflect the number of patients without confirmation of VF. The number of patients with syncope were 19 of $48(40 \%)$ in OMI, 5 of $18(28 \%)$ in DCM and 6 of $43(14 \%)$ in IVT. Syncope occurred significantly more often in OMI than in DCM or IVT. VF was confirmed in 14 of 19 patients with syncope in OMI, in 3 of 5 in DCM and 1 of 6 in IVT. The incidence of VF was significantly higher in OMI than in IVT. Although there was a patient with VF in IVT, the VF did not occur spontaneously, but was induced by a disopyramide injection.

Figure 5 shows the comparison of VTRR'm between groups with and without syncope in OMI, DCM and IVT. VTRR'ms in OMI patients with and without syncope were $0.35 \pm 0.07$ sec (mean $\pm S D)$ and $0.42 \pm 0.10$ sec, respectively $(\mathrm{p}<0.05)$. VTRR'ms in DCM patients with and without syncope were $0.43 \pm 0.10 \mathrm{sec}$ and $0.42 \pm 0.10 \mathrm{sec}$, respectively (NS). VRRR'ms in IVT patients with and without syncope 
TABLE I COMPARISON OF CI, PI, VI AND QTc BETWEEN GROUPS WITH AND WITHOUT SYNCOPE IN OMI, DCM AND IVT

\begin{tabular}{|c|c|c|c|c|}
\hline , & $\begin{array}{c}\text { Electrocardiographic } \\
\text { predictive parameters for } \\
\text { serious ventricular } \\
\text { arrhythmias }\end{array}$ & $\begin{array}{l}\text { Syncope } \\
(m \pm S D)\end{array}$ & $\begin{array}{c}\text { Non-syncope } \\
\cdot(m \pm S D)\end{array}$ & Significance \\
\hline \multirow{4}{*}{$\begin{array}{l}\text { Old myocardial } \\
\text { infarction }(O M I)\end{array}$} & $C I$ & $0.48 \pm 0.12 \mathrm{sec}$ & $0.57 \pm 0.18 \mathrm{sec}$ & $N S$ \\
\hline & $P I$ & $1.17 \pm 0.25$ & $1.40 \pm 0.42$ & $N S$ \\
\hline & $V I$ & $0.85 \pm 0.51$ & $0.57 \pm 0.21$ & $N S$ \\
\hline & $Q T c$ & $0.44 \pm 0.04$ & $0.46 \pm 0.08$ & $N S$ \\
\hline \multirow{4}{*}{$\begin{array}{l}\text { Dilated cardiomyopathy } \\
(D C M)\end{array}$} & $C I$ & $0.43 \pm 0.03 \mathrm{sec}$ & $0.52 \pm 0.15 \mathrm{sec}$ & $N S$ \\
\hline & $P I$ & $1.32 \pm 0.48$ & $.1 .33 \pm 0.29$ & $N S$ \\
\hline & $V I$ & $0.65 \pm 0.43$ & $0.56 \pm 0.15$ & $N S$ \\
\hline & $Q T_{c}$ & $0.43 \pm 0.03$ & $0.44 \pm 0.05$ & $N S$ \\
\hline \multirow{4}{*}{$\begin{array}{l}\text { Idiopathic ventricular } \\
\text { tachycardia }(I V T)\end{array}$} & $C I$ & $0.39 \pm 0.07 \mathrm{sec}$ & $0.46 \pm 0.10 \mathrm{sec}$ & $N S$ \\
\hline & $P I$ & $1.18 \pm 0.25$ & $1.30 \pm 0.41$ & $N S$ \\
\hline & $V I$ & $0.57 \pm 0.14$ & $0.61 \pm 0.17$ & $N S$ \\
\hline & $Q T_{C}$ & $0.41 \pm 0.04$ & $0.43 \pm 0.05$ & $N S$ \\
\hline
\end{tabular}

CI: coupling interval, PI: prematurity index

$V T$ : vulnerability index, $Q T c: Q T / \sqrt{R R}$

A

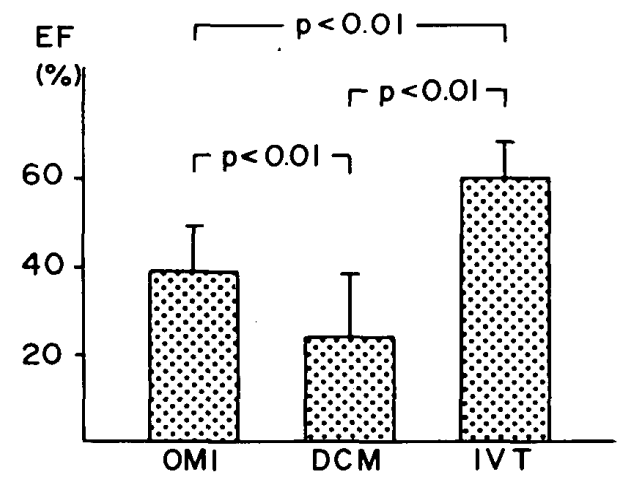

B

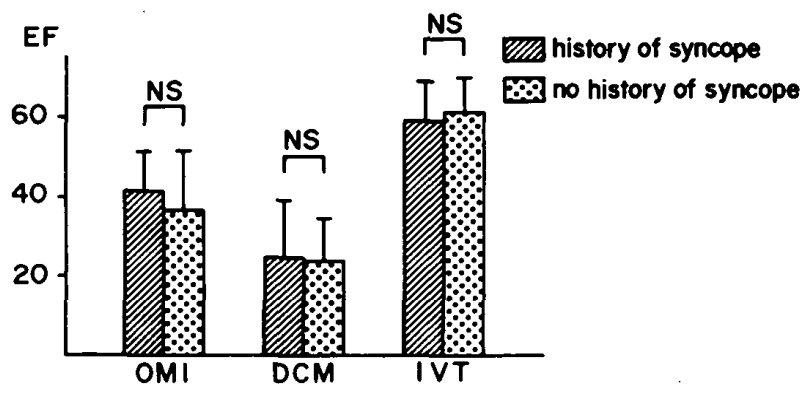

Fig.6. A: Comparison of ejection fraction (EF) determined by radionuclide "ventriculography among old myocardial infarction (OMI), dilated cardiomyopathy (DCM) and idiopathic ventricular. tachycardia (IVT), B: Comparison of EF between groups with and without syncope in OMI, DCM and IVT. were $0.27 \pm 0.04 \mathrm{sec}$ and $0.41 \pm 0.10 \mathrm{sec}$, respectively $(\mathrm{p}<0.01)$. Sustained VT, which was not followed by VF, was observed during ECG monitoring in 7 of 20 OMI patients with syncope. Four of the 7 patients with sustained VT did not have any loss of consciousness during the sustained VT and had comparatively non-rapid VTs with a mean VTRR'm of $0.37 \pm 0.03 \mathrm{sec}$. Three of 37 IVT patients without syncope had sustained VTs during ECG monitoring and also had non-rapid VTs with a mean VTRR'm of $0.40 \pm 0.04 \mathrm{sec}$.

In Table 1, comparisons of CI, PI, VI and QTc between groups with and without syncope in OMI, DCM and IVT are shown. There were no significant differences in the above parameters between the two groups in any of the underlying disease.

Figure 6A shows a comparison of the mean EF among OMI, DCM and IVT. EF in DCM was significantly lower than EF in OMI or IVT $(\mathrm{p}<0.01$ in each). EF in OMI was lower than that in IVT $(p<0.01)$. Figure $6 \mathrm{~B}$ shows the comparison of EFs between patients with and without syncope in OMI, DCM and IVT. No significant differences of EFs were found between the patients with and without syncope in any cate- 
gories of the underlying disease.

\section{DISCUSSION}

Cardiac syncope is one of the most serious problems associated with VT. Johansson ${ }^{7}$ reported that 8 out of the 16 patients with syncope, having electrocardiograms recorded during the attacks, showed ventricular tachyarrhythmias. Parkinson et al ${ }^{11}$ summarized electrocardiographical findings during the unconsciousness state of a Stokes-Adams attack in 8 patients at their hospital and 56 other reported cases. As a result, out of a total of 64 patients, 4 showed VT followed by ventricular standstill, 15 showed high VT (high VT means a rate between 200-500) usually also with VF followed by ventricular standstill, 13 showed high VT, or VF both, without ventricular standstill and the remaining 32 showed ventricular standstill alone or extreme bradycardia with complete heart block.

However, there have been very few prior studies on the comparison of the incidences of syncope and VF between VT patients with and without overt underlying disease. The relationships between the direct cause of syncope and the electrocardiographic characteristics of VT has not been commonly reported!1,12 OMI and DCM are wellknown underlying diseases associated with syncope or sudden cardiac death?-9 With respect to connections among syncope, VT and sudden cardiac death, Kapoor et $\mathrm{al}^{9}$ reported that patients with VT during Holter monitoring, who had experienced syncope, had a higher incidence of sudden death when compared to patients with rare VPCs $(18.7 \%$, vs $4.0 \% ; \mathrm{p}<0.0001)$. In addition, the finding of frequent or paired VPCs in patients with syncope had also important clinical impact on sudden cardiac death which are similar to the findings of VT.

In the present study, 9 out of 19 OMI patients with syncope had sudden death $30.7 \pm 44.9$ months (mean \pm SD) after the Holter monitoring and one or more VFs had been confirmedd in 8 of the 9 patients with sudden cardiac death. Two of the 5 DCM patients with syncope had sudden death $29.7 \pm 42.7$ months after the Holter monitoring and VF had been confirmed in both patients before the death. However, none of 6 IVT patients with syncope had sudden death.

Therefore, no sudden cardiac death would be predicted in patients with IVT even if such patients had syncope associated with VT. Actually, there have been many papers which reported good results of no underlying disease with ventricular premature ectopy with respect to sudden cardiac death, even if ventricular tachycardia or ventricular fibrillation were obsereved $4,5,6,13,14$ This study added a new finding of good results of IVT patients with respect to sudden cardiac death even if such patients would have or had experienced syncope associated with VT.

The incidence of syncope in OMI was significantly higher than that in DCM or IVT. The main cause of syncope in patients with OMI was thought to be VF, because VFs were confirmed by electrocardiograms in the majority of OMI patients with syncope $(73.7 \%)$. On the contrary, only one of the 6 IVT patients with syncope experienced VF $(16.7 \%)$. The VF of the patient did not occur spontaneously, but was induced by a disopyramide injection. Therefore, it seems that there is a difference in the direct cause of syncope between OMI and IVT.

Parkinson et al ${ }^{11}$ stated that low ventricular tachycardia (up to 160) dose not produce unconsciousness, but it provokes the subsequent ventricular standstill that dose produce it, and that high ventricular tachycardia and fibrillation (200-500) produce unconsciousness.

Hamer et al ${ }^{12}$ reported that 20 out of 40 patients having organic heart disease, 34 of whom had coronary artery disease and the remaining 6 cardiomyopathy, had syncope during sustained VT in the programmed electrical stimulation (PES) study. During PES, syncope was related to the rapidity of VT (syncope: $253 \pm 37 \mathrm{bpm}$; non-syncope: $193 \pm 24 \mathrm{bpm}, \mathrm{p}<0.001)$ and was a consequence of the degree of hypotension induced (50 $\mathrm{mmHg}$ mean arterial pressure). However, impaired resting ventricular function was not an important determinant of syncope.

In this study, OMI and IVT patients with syncope had significantly shorter mean VT cycle length (VTRR'm), which means higher VT rates, than those with no syncope 
$(\mathrm{p}<0.05$ and $\mathrm{p}<0.01$ in each). In particular, the rapidity of VT in IVT patients with syncope was high as compared to those without syncope (syncope: $228 \pm 26 \mathrm{bpm}$ vs non-syncope: $148 \pm 29 \mathrm{bpm}$ ). There were no significant differences in the EF between syncope and non-syncope group in any of the underlying diseases.

Therefore, our results suggest that syncope in IVT is based on an excessively low cardiac output caused by very rapid VT. The cause of syncope in OMI patients is mainly VF and subsequently the very rapid VT. Low EF would not be the cause of syncope from the data of Hamer et al or from our data.

However, it remains unkown from this study why VTs in IVT patients dose not develop into VF, while VTs in OMI patients were predisposed to develop into VF. CI, PI, VI, or QTc of VT would not be important predictors for syncope, since there were no significant differences in the values of CI, PI, VI andQTc between groups with and without syncope in this study.

\section{REFERENCE}

1. VISMARA LA, AMSTERDAM EA, MASON DT: Relation of ventricular arrhythmias in the late hospital phase of acute myocardial infarction to sudden death after hospital dischange. Am J Med 59: 6, 1975

2. BIGGER JTJr, WELD FM, ROLNITZKY LM: Prevalence, characteristics and significance of ventricular tachycardia ( 3 or more complexes) detected with ambulatory electrocardiographic recording in the late hospital phase of acute myocardial infarction. Am J Cardiol 48: 815, 1981

3. BIGGER JT, FLEISS JL, KLEIGER R, MILLER VP, ROLNITZKY LM: The Multicenter Postinfarction Research Group: The relationships among ventricular arrhythmias, left ventricular dysfunction, and mortality in the 2 years after myocardial infarction. Circulation 69: 250, 1984

4. MONTAGUE TJ, McPHERSON DD, MacKENZIE BR, SPENCER CA, NANTON MA, HORACER BM: Frequent ventricular ectopic activity without underlying cardiac disease: analysis of 45 subjects. Am J Cardiol 52: 980, 1983

5. KENNEDY HL, WHITLOCK JA, SPRAGUE MK, KENNEDY LJ, BUCKINGHAM TA, GOLDBERG RJ: Long-term following of asymptomatic healthy subjects with frequent and complex ventricular ectopy. $N$ Engl J Med 312: 193, 1985

6. TANABE T, GOTO Y: Long-term prognostic assessment of ventricular tachycardia with respect to sudden death in patients with and without overt heart disease. Jpn Circ J 53: 1557, 1989

7. JOHANSSON BW: Adams-Stokes Syndrome: A review and follow-up study of forty-two cases. Am J Cardiol 8: 76, 1961

8. KAPPOR WN, KARPF M, WIEAND S, PETERSON JR, LEVEY GS: A prospective evaluation and follow-up of patients with syncope. N Engl J Med 309(4): 197, 1983

9. KAPPOR WN, CHA R, PETERSON JR, WIEAND HS, KARPF M: Prolonged electrocardiographic monitoring in patients with syncope. Am J Med 82: 20, 1987

10. HAN J, GOEL BG: Electrophysiologic precursors of ventricular tachyarrhythmias. Arch Intern Med 129: 749, 1972

11. PARKINSON J, RAPP C, EVANS W: The electrocardiograms of the Stokes-Adams attack. $\mathrm{Br}$ Heart J 3: 171, 1941

12. HAMER AWF, RUBIN SA, PETER T, MANDEL WJ: Factors that predict syncope during ventricular tachycardia in patients. Am Heart $J$ 107(5): 997, 1984

13. TRAPPE HJ, BRUGADA P, TALAJIC $M$, BELLA PD, LEZAUN R, MULLENEERS R, WELLENS HJJ: Prognosis of patients with ventricular tzchycardia and ventricular fibrillation: Role of the underlying etiology. J Am Coll Cardiol 12: 166, 1988

14. BELHASSEN B, SHAPIRA I, SHOSHANI D, PAREDES $A$, MILLER $H$, LANIAD S: Idiopathic ventricular fibrillation: inducibility and beneficial effects of class I antiarrhythmic agents. Circulation 75: 809, 1987 